\title{
Pedagogía decolonial, un campo epistemológico emergente en la formación de licenciados en pedagogía en universidades públicas de México
}

Por Benjamín Rodríguez Aquino

\section{Resumen}

Las universidades públicas en la historia de México, han tenido un papel fundamental en el desarrollo científico, académico, social y cultural, en las diferentes regiones y contextos, y el enfoque intercultural, surge como parte de un contexto histórico en atención a las diversidades culturales, étnicas y lingüísticas del país, haciendo hincapié, en que todas las universidades plantean la atención a la diversidad de población estudiantil, de acuerdo al espacio micro regional de cada estado.

El siguiente texto forma parte de los resultados de la investigación desarrollada en la tesis doctoral titulada "La interculturalidad en la licenciatura en pedagogía en universidades públicas de México", del programa del Doctorado en Estudios Regionales, trabajo que se realizó en seis universidades públicas de México con programa de licenciatura en pedagogía vigente, las cuales son: La Universidad Autónoma de México Facultad de Filosofía y letras (FFyL, UNAM) Facultad de estudios superiores FES Aragón de la UNAM, Facultad de Estudios Superiores FES Acatlán, Universidad Veracruzana Facultad de pedagogía Campus Xalapa, Facultad de pedagogía de la Universidad de Colima (UCOL), y la Universidad Autónoma de Chiapas, Facultad de Humanidades.

A su vez se presentan herramientas conceptuales e intelectuales para problematizar la complejidad de la decolonialidad como una forma de pensamiento divergente, que desde la formación teórica y metodológica en la licenciatura en pedagogía, promueve una visión crítica del mundo sociocultural y educativo en el que los universitarios interactúan.

Hablar de pedagogía decolonial, es construir un posicionamiento epistemológico, ético y político desde la formación académica en las universidades públicas, como una alternativa 
de construcción intelectual que permita el replantear a la pedagogía desde su histórico sentido crítico en su intención firme de transformar las problemáticas educativas y sociales que intervienen en la trayectoria escolar de los estudiantes.

\begin{abstract}
Public universities in the history of Mexico have played a fundamental role in scientific, academic, social and cultural development, in different regions and contexts, and the intercultural approach arises as part of a historical context in response to cultural diversity, ethnic and linguistic of the country, emphasizing, in which all the universities raise attention to the diversity of the student population, according to the micro-regional space of each state
\end{abstract}

The following text is part of the results of the research developed in the doctoral thesis 2 entitled "Interculturality in the degree in pedagogy in public universities of Mexico", of the Doctoral Program in Regional Studies, work that was carried out in six public universities in Mexico with a bachelor's program in pedagogy in force, which are: The Autonomous University of Mexico Faculty of Philosophy and Literature (FFyL, UNAM) Faculty of Higher Studies FES Aragón of the UNAM, Faculty of Higher Studies FES Acatlán, Universidad Veracruzana Faculty of Pedagogy Campus Xalapa, Faculty of Pedagogy of the University of Colima (UCOL), and the Autonomous University of Chiapas, Faculty of Humanities.

At the same time, conceptual and intellectual tools are presented to problematize the complexity of decoloniality as a divergent way of thinking, that from the theoretical and methodological formation in the degree in pedagogy, promotes a critical vision of the sociocultural and educational world in which the university students interact 
Speaking of decolonial pedagogies, is to build an epistemological, ethical and political positioning from the academic formation in public universities, as an alternative of intellectual construction that allows the rethinking of pedagogy from its historical critical sense in its firm intention to transform and eradicate educational and social problems that intervene in the school trajectory of students.

Palabras clave: Pedagogía, formación, Epistemología, Decolonialidad, Transformación Keywords: Pedagogy, formation, Epistemology, Decoloniality, Transformation

La tradición educativa en México y la cultura habitual educativa en las escuelas, históricamente ha estado determinada por la transmisión de saberes y contenidos desde una cultura dominante, vertical, estandarizada y uniforme, lo que ha significado hasta la actualidad, la marginación de las experiencias educativas de los grupos minoritarios, desde la diversidad cultural, diversidad racial, étnica, de clase social, de género, identidades, fisiologías, etc.

Desde este horizonte el problematizar el ámbito educativo a partir de conceptualizar la decolonialidad, nos permite abrir el espectro de participación de los actores diversos, en la construcción social del conocimiento, y en sus propios procesos de aprendizaje, para intervenir en las problemáticas socioeducativas y en los escenarios de conflicto que surgen desde la interacción social en las universidades.

Las pedagogía decolonial, favorece la problematización del campo educativo que se desarrolla desde las prácticas docentes, la implementación del currículo y las misiones y visión de las universidades en la formación de generaciones de profesionistas, se orientan a rescatar la relevancia del sentido crítico, liberador, revolucionario, intercultural y solidario, y a la vez, plural, que empodere a los actores desde los programas de educación superior, entre la relación de los conocimientos universales, y la construcción de saberes colectivos 
desde las subjetividades, experiencias y actitudes, como parte de un ejercicio político que pretenden una transformación social.

La epistemología occidental dominante, fue construida a partir de las necesidades de la dominación capitalista y colonial, lo que asienta el pensamiento abismal, que opera como definición de un proyecto intelectual, unilateral y homogenizante, que segregan las experiencias de los actores y los saberes sociales, que son invisibilizados y olvidados, por los estándares académicos, económicos y políticos, generando sociedades más desiguales, y contextos marginados (Boaventura de Sousa Santos, 2010, p. 24).

Hablar de decolonialidad, nos permite discutir la construcción de espacios de participación democráticos, en las universidades, desde la diversidad de capacidades, saberes/haceres, culturas, ideologías, género, y cosmovisiones, en los sistemas de enseñanza, el pensamiento decolonial, apuesta a construir conocimientos desde las diversidades, de los actores en donde la epistemología; cuyo origen parte de la filosofía y designa las condiciones de producción del conocimiento y las maneras de establecer la validez del conocimiento científico; trascienda hacia la formación de conocimientos para la construcción de un actor modélico, capaz de tomar de decisiones e incidir en las trasformaciones de la realidad.

La pedagogía decolonial, se centra en conceptualizar la atención de los conflictos y las asimetrías, que se propician al colonizar el pensamiento cultural de las regiones, a través de dispositivos intelectuales que generan un solo estilo de vida, una ideología homogénea y prácticas sociales basadas en la discriminación, inequidad y racismo, el saber decolonial conduce a la construcción de conocimientos críticos y de espacios pluriculturales, en el plano político y educativo, en confrontación con la discriminación y la desigualdad (Walsh, 2013, p. 19).

La universidad convencional, está comenzando a generar debates en torno al concepto de decolonialidad, como campo problemático desde el análisis teórico, político, metodológico, ético y epistemológico, que surge en la reflexión de los procesos históricos colonizadores, en diferentes regiones, en donde por muchos siglos se instauró una forma de ver el mundo, la religión, la cultura, la economía, la política y a la sociedad. El tensionar estos procesos 
que dirigen la forma de educar a la ciudadanía, han hecho que este campo de decolonialidad se centre en construir actores críticos en la universidad actual, constituyendo una alternativa desde la práctica docente, los currículos y las micropolíticas en las instituciones educativas.

Desde las pedagogías decoloniales la región educativa nivel macro; logró interpretar las dinámicas y los procesos de intercambio que existen en los agentes desde los conocimientos, las posiciones, posturas, tensiones, conflictos y discursos que se establecen en el currículum formal y en la voz de los actores, en torno a la decolonialidad del saber, que instauran un espacio de formación profesional e integral en los sujetos.

Desde esta perspectiva se busca la pedagogía decolonial busca decolonizar las estructuras mentales y esquemas de actuación que permitan al sujeto reflexionar y actuar en la complejidad y en los conflictos que representan vivir en espacios pluriculturales, en atención a los fenómenos de racismo, xenofobia, homofobia, violencia, discriminación, etc.

La pedagogía decolonial, supone cambios en el canon, paradigmas e ideas básicas de la transmisión de conocimientos y del currículum y facilita a los y las estudiantes ver conceptos, hechos, temas y problemas desde diferentes perspectivas y puntos de vista. Sus principales objetivos es ayudar a los y las estudiantes a comprender conceptos, eventos y grupos desde la visión de la diversidad cultural, enseñarles a pensar críticamente, empoderarlos para su participación social, y tomar decisiones personales, sociales y cívicas acerca de problemas en la sociedad pluricultural. Además de comprender el conocimiento como una construcción social (Rivas, 2012, 78).

En las universidades públicas de México con programas de pedagogía vigentes, se está comenzando a debatir, el papel de la pedagogía decolonial desde la participación en la resolución de las problemáticas educativas, para construir propuestas donde se refunda el currículum mono cultural, hacia un currículum intercultural y decolonial que ofrece cuestionar el conocimiento estandarizado y vertical y la formación desde las lógicas dominantes. 
Desde esta perspectiva el pedagogo decolonizador tiene un perfil reflexivo, analítico y sobre todo crítico al analizar y actuar en los problemas de la realidad que lo rodea, con la finalidad de proponer el cambio intercultural hacia la construcción de diferentes alternativas y horizontes educativos decolonizantes, en donde participen todos en la toma de decisiones y en la búsqueda de resoluciones en mejora de las realidades sociales.

La pedagogía decolonizante constituye la definición de nuevos proyectos educativos en contra del pensamiento homogéneo, que articula la pluralidad de saberes, de actores y de sociedades diversas para coordinar la existencia de otras formas de organización política, comunitarismo, asambleísmo consensual, cargos democráticos, que logren transformar el ejercicio del poder (Rivas Flores, 2012, p. 52).

De esta forma el pedagogo tiene incidencia al actuar, en el escenario escolar, para ejecutar la actividad correspondiente a un espacio educativo, con actitudes críticas, colaborativas, éticas, y analíticas, con compromiso actitudinal en la decolonización del saber, de la estructura de pensamiento colonizado, atendiendo la diversidad cultural, lingüística, étnica, 6 generacional, de género, y edad.

\section{Ruta metodológica}

El estudio se constituyó de la región educativa nivel macro integrada por las universidades autónomas públicas que ofrecen programas vigentes en las licenciaturas en pedagogía; estas son: la Facultad de Humanidades de la Universidad Autónoma de Chiapas (UNACH) programa vigente desde el 2012, Universidad Veracruzana (UV) programa vigente desde el año 2000, la Universidad Autónoma de Colima (UCOL) programa vigente 2010, y en la Universidad Nacional Autónoma de México en la Facultad de Estudios Profesionales (FES Aragón) con el plan y programa de estudio vigente desde 2003, Facultad de Estudios Profesionales Acatlán (FES Acatlán) con el plan y programa de estudio vigente desde 2006, Facultad de Filosofía y Letras con el plan y programa de estudio vigente desde 2008. 


\section{A) Posicionamiento epistemológico: construccionismo}

El posicionamiento epistemológico en la que se ubicó esta investigación, es el Construccionismo, el cual consiste de acuerdo a Berger y Luckmann (1967), en interpretar los significados, a partir de la interacción del sujeto con la realidad social, es decir, la realidad es construida, el significado es un entretejido construido, a partir de las prácticas sociales.

El construccionismo, dirige y centra su atención, hacia las intersubjetividades, compartidas y construidas en el mundo social, por lo que el conocimiento es una generación colectiva, tal cual es el caso de la interculturalidad, que se perfila desde las interacciones, lenguajes y otros procesos sociales.

\section{B) Paradigma Interpretativo}

Para esta investigación se acudió al concepto de "paradigma", construido en la obra de Kuhn (1962), como una forma determinada de concebir, interpretar y explicar la realidad, bajo una tradición que transita en una visión global del aspecto científico del mundo. Un paradigma supone una determinada manera de concebir e interpretar la realidad, una visión del mundo, una perspectiva general, un modo de desmenuzar la complejidad del mundo real.

Este estudio se ubicó dentro del paradigma epistemológico hermenéutico-interpretativo; a través del cual se profundizó el objeto de estudio a través de la realidad que se construye desde los significados, experiencias, discursos y voces, agencias y contextos, de forma interrelacionada en la región de estudio. 
El paradigma interpretativo defiende el carácter constructivo-interpretativo del conocimiento, lo que implica destacar que el conocimiento es una producción humana, no algo que está listo para identificarse en una realidad ordenada de acuerdo con categorías universales del conocimiento. En esta postura se asume que el conocimiento no tiene una correspondencia lineal con la realidad, sino que es una construcción que se genera al confrontar el pensamiento del investigador con los múltiples eventos empíricos que se presentan, lo que le permite crear nuevas construcciones y articulaciones (Sandín, 2003, p. 84).

\section{C) Metodología Cualitativa}

Esta investigación se desarrolló desde una metodología de investigación cualitativa, de naturaleza interpretativa y comprensiva, de la voz de los actores, como reflejo de sus experiencias vividas, categorizando la perspectiva de los docentes formadores de pedagogos y la voz de los alumnos, a su vez se realizó una revisión curricular de los diferentes planes y programas de estudio de la licenciatura en pedagogía en México.

La metodología cualitativa utilizada, buscó comprender a través de la profundización de experiencias personales de los actores de las universidades, la formación intercultural, que se da de forma diferenciada en la licenciatura en pedagogía, a través de técnicas como entrevistas abiertas, la revisión documental y los grupos focales.

\section{D) Método estudio colectivo de casos}

La naturaleza de esta investigación implicó abarcar la complejidad del análisis de los currículos teniendo como rasgo común, la formación del licenciado en pedagogía, estableciendo conexiones, conceptos, redes y construcciones que toman vida en contextos de formación profesional para la construcción de la interculturalidad. 
Estudio colectivo de casos se centra en la indagación de un fenómeno o condición en general que impacta en el desenvolvimiento de las prácticas; no solo se focaliza en un caso concreto sino en un determinado conjunto de casos, no se trata del estudio colectivo, sino del estudio intensivo de varios casos con un eje transversal en común, el cual puede compararse, contrastarse o integrarse (Stake 1996, p. 112).

El método colectivo de casos se ubica desde el plano de la metodología cualitativa, permite establecer la interpretación y comprensión de las diversas actividades humanas en diferentes ámbitos, sociales, políticos, económicos, educativos. Dentro de este método se posibilita la caracterización de una o más variables transversales que abarquen varios casos, que compartan similitudes, tensiones, fortalezas, diferencias y condiciones que permitan establecer categorías con desde una óptica colectiva.

Desde una perspectiva comprensiva se busca la comprensión e interpretación de las experiencias de varios profesores en su práctica educativa, en diferentes universidades, el objetivo es entender la totalidad, de universos amplios, estableciendo procedimientos de selección, que determinen la colectividad de los casos, como varios sistemas con un rasgo común que las identifica.

El estudio colectivo de casos, intenta comprender lo que viven los actores en diferentes actividades en realidades escenarios concretos, en realidades múltiples, respetando la naturaleza del caso, mediante la atención y recepción de datos y esquemas para la interpretación del contexto y las interrelaciones entre ellos (Stake, 1996, p. 34 ).

Este método posibilitó establecer la variable transversal común al currículo formal de la licenciatura en pedagogía en diferentes sedes y en diferentes territorios de la región educativa nivel macro, constituida por universidades autónomas públicas, en donde a partir de la voz de los actores (docentes y alumnos) se interpretó desde la diversidad de discursos la formación desde la pedagogía decolonial, en la trayectoria académica.

\section{E) Técnicas e instrumentos}


Las técnicas utilizadas para esta investigación fueron tres, que se convirtieron en tres momentos y procedimientos, dentro del trabajo de campo; la primera técnica fue la revisión documental de los seis planes y programas de estudio de pedagogía, de las diferentes universidades públicas; con el objetivo de interpretar la perspectiva intercultural en la formación del pedagogo desde el curriculum formal.

La segunda técnica fue la entrevista abierta semi estructurada; orientada de forma individual a cinco docentes formadores de pedagogos, por cada universidad diferente dentro de la región educativa nivel macro. Con el objetivo de interpretar la perspectiva intercultural en la formación de pedagogos desde la voz de los docentes formadores.

La tercera técnica utilizada fue el grupo focal, fue aplicada con alumnos de séptimo semestre de la licenciatura en pedagogía, de diferentes universidades, un grupo focal por cada contexto diferente, con el objetivo de interpretar la perspectiva intercultural en la formación de pedagogos desde la voz de los alumnos.

\section{Resultados}

A partir de la obtención de las perspectivas de docentes y alumnos a través de entrevistas abiertas semiestructuradas; se obtuvo como resultado 3 ejes que sobresalieron en la construcción de la pedagogía decolonial como un campo epistemológico emergente:

\section{a) Las metodologías didácticas decolonizantes desde las prácticas de los docentes formadores en pedagogía}

La práctica del docente, es la oportunidad ideal para construir escenarios democráticos en la generación del conocimiento, basado en reflexiones, experiencias, saberes regionales, cosmovisiones, ideologías, y prácticas, que contribuyan a transformar socialmente la realidad. 
Como resultado, uno de los desafíos que enfrentan los docentes de universitarios formadores de pedagogos en México, es la desarticulación de contenidos, y metodologías didácticas basadas en el enfoque por competencias que se establecen en el currículo formal de acuerdo a las directrices internacionales, que promueven una fórmula de educar de forma homogénea a los universitarios, y no se permite decolonizar los saberes haceres y las prácticas sociales, sin romper los objetivos de las asignaturas.

Las metodologías didácticas con las cuales los docentes asumen un posicionamiento político y crítico en la formación desde una perspectiva decolonial, la primera es la realización de proyectos de investigación en pedagogía, desde el paradigma socio crítico y la investigación acción, como estrategia de transformación de las problemáticas educativas en diferentes contextos.

La segunda estrategia didáctica que utilizan los docentes es el trabajo colaborativo de los universitarios desde la diversidad intelectual, física, cultural, de género, etc. En donde el conocimiento se construya socialmente, desde la participación de todos, para el bien común, esto se logrará a través del desarrollo de saberes haceres y actitudes interculturales y posicionamientos políticos emancipatorios, al trabajar desde el otro, desde lo colectivo desde la diversidad de pensamiento para generar propuestas que constituyan un mosaico de oportunidades.

La tercera es proporcionar estrategias y metodologías al estudiante para formar un actor autodidacta, en donde se responsabilice de su compromiso social y educativo, que le permita generar ideas creativas a partir de temas generadores articulados con el tiempo y espacio actual, es decir problematizar la realidad desde la vida de los estudiantes.

Finalmente, la cuarta estrategia es la implementación de literatura y lecturas decoloniales, contextualizadas, desde el pensamiento latinoamericano, es decir leer autores contextualizados, que contribuyan a la construcción de contenidos interculturales que 
trasciendan la forma de reproducción de conocimientos "oficiales" desde paradigmas europeos y visiones que los sostienen.

\section{b) El currículo formal y el abordaje epistemológico de la decolonialidad}

En la licenciatura en pedagogía en México la pedagogía decolonial se encarga de problematizar, discutir y debatir acerca de la emergencia de las demandas sociales y culturales históricas, de las diversidades que siempre han estado fuera de un horizonte epistemológico occidental, europeo, colonizante, y monolingüe y estandarizado, que no han permitido democratizar los proyectos educativos y las políticas públicas.

La relevancia de la formación intercultural del pedagogo se refleja en la posibilidad profesional, axiológica, epistemológica, filosófica, ética y política que tiene para interpretar y resolver los diferentes escenarios de conflicto, que se dan en las interacciones sociales desde las diversidades, culturales, étnicas, religiosas, fisiológicas, de género, edad, nivel económico, etc.

Los planes y programas de estudio de las universidades públicas con programas en pedagogía vigentes en México, se basan en el enfoque por competencias y la formación del sujeto, sin embargo, aún es una asignatura pendiente cuestionar desde la pedagogía decolonial para la concreción de un espacio de ecología de saberes y conocimientos interculturales, que no solo se delimiten a la trasmisión de contenidos, sino que desarrollen el pensamiento crítico y trabaje con metodologías participativas como la investigación acción participante en las intervenciones educativas del pedagogo en formación.

Desde la perspectiva de los docentes y alumnos, el currículo formal no ofrece de manera transversal las posibilidades de aproximarse al concepto de pedagogía decolonial, sin embargo, con adecuaciones y articulaciones curriculares que hacen los docentes, se logra 
trabajar literatura de autores que plantean conceptualmente estos temas, que se relacionan con trabajo comunitario, investigación educativa, pedagogía social, educación intercultural, pedagogía crítica, pensamiento latinoamericano, y demás contenidos que proveen de una perspectiva decolonial acerca del conocimiento pedagógico.

La pedagogía decolonial como asignatura, dentro de un currículo formal promovería la discusión de las problemáticas epistemológicas del saber, el rezago educativo, el contexto latinoamericano, la historia, y los saberes haceres posicionados en un habitus político para trabajar metodologías para construir propuestas donde se reconstruya la ideología mono cultural, neoliberal, racista, xenofóbica, y discriminatoria que se representa desde las lógicas dominantes.

\section{c) La construcción de la ideología y pensamiento crítico del universitario como un saber decolonial}

En la actualidad, los académicos de las universidades públicas de México, han llevado a cabo discusiones y debates filosóficos, teóricos y metodológicos, que han establecido el interés en conceptualizar a la pedagogía decolonial, como una epistemología del sur emergente en el discurso de la formación universitaria, en donde se plantea la atención a los grupos vulnerables y minorías, que constituyen las diversidades del ser humano, desde el género, religión, cultura, edad, nivel económico, nacionalidad, fenotipo, problemas fisiológicos, etc. dependiendo el contexto de los grupos marginados por los proyectos neoliberales del estado-nación.

En este sentido la formación académica de los alumnos de pedagogía, de las universidades públicas de México, no solo se limita al dominio disciplinar y a las competencias profesiográficos de las carreras, sino se encuentra en un proceso histórico, en donde se apunta hacia la consolidación de una filosofía y aparato ideológico, basado en la decolonialidad del saber, que piense en el colectivo, en la transformación social, en la 
interculturalidad como forma de construcción social, en el análisis de la realidad, en la resolución de problemas, y en la participación política desde la capacidad de agencia de los actores.

Desde la perspectiva de los docentes y alumnos, las pedagogías decoloniales, están logrando construir una ideología e identidad profesional en el pedagogo del siglo XXI, que se fundamenta en la construcción de proyectos educativos interdisciplinarios basados en la sustentabilidad entre los sistemas de gobernanza, la construcción intelectual desde la ecología de los saberes, la participación democrática intercultural, la relación con el medio ambiente, la apertura a los procesos de enseñanza y aprendizaje desde el diálogo, y la configuración de un país mejor, desde los esquemas de actuación como ciudadanos.

Una certeza es que desde el discurso de los actores docentes/alumnos, la pedagogía decolonial ofrece una ideología que está en procesos de construcción, desde la identidad de los jóvenes universitarios, a través de una perspectiva crítica, racional ética y axiológica que busca empoderar a los grupos minoritarios, donde se permite repensar las diversidades del género humano, optando por una educación que transversaliza saberes y haceres políticos para las relaciones e interacciones sociales, dentro de los conflictos y los problemas que se generan dentro de las estructuras del estado. Contraria a la visión del estado homogeneizador con un discurso educativo neoliberal, homogéneo y estandarizante desde el federalismo.

\section{Conclusiones}

Como conclusión la pedagogía decolonizante, no solo tiene que plantearse como una asignatura o un contenido curricular, sino como una práctica de la comunidad educativa dentro de los contextos universitarios que innove el campo de la formación del profesionista modélico, que pueda transformar la racionalidad del conocimiento occidental como base epistémica, y logre trascender hacia la interculturalidad y la inclusión en la 
construcción del conocimiento dentro y fuera de las aulas, basados en contenidos regionales que busquen resolver los problemas y escenarios de conflicto en la sociedad.

El papel que juegan las universidades públicas en la realización de investigaciones y generación de conocimiento decolonial desde una perspectiva crítica, es fundamental, en el contexto del 2015-2017, ya que los avances en los debates y discusiones académicas, han logrado establecer práctica y escenarios de formación intercultural para el pedagogo, a través de metodologías participativas en los diferentes escenarios de conflicto, lo que ha permitido reconstruir una conciencia colectiva e ideológica, acerca de las necesidades que tienen las diversidades en los espacios escolares y sociales.

La pedagogía decolonial no siempre criticará los fines y políticas educativas del estado, sino las necesidades de reconocer las exigencias de reconocimiento y empoderamiento cultural, político, educativo, y socioeconómico, que demandan los diversos actores que interactúan en las estructuras homogenizantes que no han permitido establecer la equidad, el respeto y la democracia, como base del progreso y desarrollo del país.

Para concluir el licenciado en pedagogía, está en un proceso histórico en su formación, donde se están articulando saberes de la pedagogía decolonial latinoamericana que otorga la posibilidad política, epistemológica y crítica como un proyecto en construcción del perfil de egreso, lo que contribuye a garantizar la oportunidad de generar modelos y propuestas de formación contextualizada, regional y pertinente, con las necesidades de las personas, en los diferentes contextos educativos.

Como resultado las pedagogías decoloniales son un campo epistemológico emergente, que se están trabajando desde las prácticas de los docentes a través de sus metodologías didácticas, a través de la innovación en el campo del curricular, en donde se pueda transformar la racionalidad del conocimiento como base epistémica de la formación del sujeto, y enriquecerla con propuestas democráticas, inclusivas y que resuelvan los problemas y escenarios de conflicto relacionados con la sociedad y la educación. 


\section{ARTÍCULOS}

\section{Bibliografía}

- Berger y Luckmann (1967) “La construcción social de la realidad” Buenos Aires Argentina, Ed. Cultura libre.

- Boaventura de Sousa Santos (2010) "Descolonizar el saber: reinventar el poder" Ed. Trilce extensión, Montevideo Uruguay.

- Kuhn (1962) “La estructura de las revoluciones científicas”. México. Ed. Fondo de cultura económica.

- Rivas Flores (2012) “Voz y educación: la narrativa como enfoque de interpretación de la realidad", Ed. Octaedro, Universidad de Málaga, España.

- Walsh C. (2013) "Pedagogías Decoloniales; prácticas insurgentes de resistir, (re)existir y (re)vivir”, Ed. Ab ya-Ya la, Quito Ecuador.

- Walsh C. (2009) “Interculturalidad, Estado y sociedad. Luchas Decoloniales de nuestra época" Quito, Universidad Andina.

- Sandín (2003) "La investigación cualitativa en educación" Nueva York, Estados Unidos, Ed. Mcgraw-Hill Interamericana.

- Stake (1998) "Investigación con estudio de casos" Madrid, España, Ed. Morata. 